\title{
Transition
}

Mein Kollege Chris war definitiv unkonzentriert. Seit einigen Tagen waren wir im Humanitarian Hub des UNMISS-Camps nahe Bentiu zu Gast. Bentiu lag zum Zeitpunkt unserer Reise in Northern Liech State, einem von zu diesem Zeitpunkt 32 Gliedstaaten. Heute ist es wieder die Hauptstadt von Unity State. Wir waren hier, um Interviews und Fokusgruppen-Diskussionen zu führen. Geplant waren Gespräche mit verschiedenen Teilen der Bevölkerung, in Bentiu und seiner Schwesterstadt Rubkona, sowie im größten Protection of Civilian (PoC) Camp des Landes. In dem Camp, direkt neben UNMISS gelegen, haben mehr als 160.000 Menschen Zuflucht gefunden. Im Zuge unseres gemeinsamen Abendessens wurde klar, dass Chris ein anderes Problem beschäftigte. Tapfer gegen die brüchige Mobilfunkverbindung ankämpfend, telefonierte er unermüdlich mit einer unerschöpflichen Reihe an Freund:innen, Verwandten und Kolleg:innen. Schnell war klar, wo das Problem lag: Es fehlte ihm an Geld.

Vor einiger Zeit wurde Chris in Juba in einen Autounfall verwickelt. Ein betrunkener Fahrer hatte ihn an einer Kreuzung abgeschossen, die Konsequenz war ein Totalschaden. Der Unfallgegner hatte sich, nicht zuletzt motiviert durch die Perspektive einer Vermeidung der südsudanesischen Bürokratie, bereiterklärt, ein gleichwertiges Auto zu beschaffen. Es handelte sich um einen Gebrauchtwagen aus Japan, verschifft über Mombasa und dann weitertransportiert über den Landweg nach Juba, via Nimule an der ugandischsüdsudanesischen Grenze. Nimule war es auch, wo das neue Auto von Chris nun stand und auf Zoll-Clearance wartete. Das Geld des Unfallgegners hatte nur für Ankauf, Transport und den Zoll im Hafen von Mombasa gereicht. Die südsudanesischen Zollgebühren, einige hundert US-Dollar, waren nicht mehr einzutreiben. Chris wiederum hatte sein letztes Gehalt von der University of Juba im vorangegangenen September ausgezahlt bekommen. Er arbei- 
tete seit fast einem halben Jahr ohne Bezahlung. Seine Geldreserven waren aufgebraucht.

Die Situation war spürbar angespannt. Es ging nicht nur um das neue Auto, die Vorfreude, den unmittelbaren Bedarf. Die Nervosität war mit einem bestimmten Datum verknüpft - dem 22. Februar 2020.

Südsudan verfügt zum Zeitpunkt unserer Forschungsreise über eine Regierung mit dem schmucklosen Akronym GoSS, das Government of the Republic of South Sudan, naturgemäß unter der Präsidentschaft von Salva Kiir Mayardit. SPLM-IO-Überläufer Taban Deng Gai fungiert als Erster Vizepräsident. Dies ist jedoch nicht die Konstellation, die im R-ARCSS-Abkommen ausgehandelt wurde. Laut Friedensvertrag soll der Vorsitzende der SPLM-IO, Riek Machar Teny, Taban Dengs Position übernehmen. Dr. Riek, wie er landläufig genannt wird, befindet sich zu diesem Zeitpunkt noch in Khartum. Er weigert sich, in Ermangelung verlässlicher Sicherheitsgarantien und einer Rückabwicklung der $32 \mathrm{zu}$ wieder 10 Gliedstaaten die für ihn vorgesehene Position in Juba einzunehmen.

Die Implementierung des R-ARCSS befindet sich nach wie vor in der »PreTransition Phase«. Diese Phase der Vortransition sollte laut Abkommen bis zur Einrichtung einer gemeinsamen Regierung zwischen den Vertragsparteien andauern. Die Basis dieser Einrichtung ist eine bis auf County-Ebene durchdeklinierte Machtteilungsformel. Ebenso vorgesehen ist die Etablierung sogenannter Necessary Unified Forces, einer gemeinsamen Armee, gebildet aus den Truppenkörpern von SPLA, SPLA-IO und einigen kleineren Vertragsparteien. Ursprünglich sind acht Monate für die Phase vorgesehen. Gemäß dem Datum der Unterzeichnung des Vertrages am 12. September 2018 hätte die Vortransition also bereits Mitte Mai 2019 abgeschlossen sein müssen. Bald hatte sich diese Deadline jedoch als vollkommen unrealistisch herausgestellt. Mehrere Verlängerungen der Pre-Transition Phase und eine Anpassung der vorgesehenen Benchmarks sind die Folge.

Fallengelassen wird etwa die Bedingung der Etablierung der Necessary Unified Forces vor Beginn der formellen Transitionsphase. Angesichts der massiven Probleme in den Cantonment Sites, in denen sich die Soldat:innen aller Truppenkörper zu Screening und Selektion für das gemeinsame Training einzufinden hatten, ist dies eine unumgängliche Entscheidung. Am Ende ist es nur mehr die konkrete Formierung des R-TGoNU - sprich die Rückkehr von Riek Machar nach Juba und der offizielle Einzug der SPLM-IO in die Übergangsregierung - die von der ursprünglichen Implementationsmatrix für die Vortransition übrigbleibt. Diese Bedingung hängt jedoch am seidenen Faden 
einer rasch ablaufenden Zeitleiste. Die Überwachungsgremien der Vortransition, die rekonstituierte Joint Monitoring and Evaluation Commission (RJMEC) und das National Pre-Transition Committee (NPTC) hatten sich auf eine letzte 100-Tages-Frist verständigt, innerhalb derer die Übergangsregierung zu bilden war.

Allein schon der Begriff einer Pre-Transition Phase verrät viel über den derzeitigen formellen Friedensprozess im Südsudan. Nicht zuletzt erschüttert er durch seine Existenz ontologische Grundannahmen. Ist so etwas wie eine Vortransition in einem Friedensprozess, der ja per definitionem ein Transitionsprozess ist, überhaupt denkmöglich? Kann das temporale Element einer Transition eingefangen oder gar angehalten werden wie eine Stoppuhr, die erst zu laufen beginnt, wenn verschiedene Grundvoraussetzungen erfüllt sind? Kann sich die Transition in dieser Weise selbst zu einer Konfliktlandschaft entwickeln, in deren Rahmen politische Aushandlung stattfindet? Die naheliegende philosophische Antwort auf diese Frage muss wohl lauten, nein, das sollte eigentlich nicht möglich sein. Eine Pre-Transition Phase in einer Post-Konflikt-Transition kann konzeptionell nicht existieren, sie wäre ein absurdes und unlogisches Konstrukt.

Mit derartigen Sichtweisen ist die von R-ARCSS induzierte Transition im Südsudan jedoch nicht zu verstehen. Vielleicht ist es eine ebenso legitime Lesart, den gesamten revitalisierten Friedensprozess als eine Nicht-Transition zu lesen. Trotz aller Erleichterung, die viele Beobachter:innen nach der Unterzeichnung des Friedensvertrages überkommen hat, ist ein genuiner Wille zum Friedensschluss kaum auszumachen. Der Vertragsabschluss war für alle Seiten zuallererst ein taktisches Manöver, die zum gegebenen Zeitpunkt bestmögliche Entscheidung zur Wahrung der eigenen Interessenlagen. Und gerade bei der SPLM-IG, die sich als Siegerin des Bürgerkriegs wähnte und immer noch wähnt, ist die Bereitschaft zum Eingehen von Kompromissen nur schwach ausgeprägt.

Eine ganze Reihe von Indizien veranschaulicht eine solche Interpretation. Neben der seltsamen Vereinbarung zu einer Vortransition ist schon die Unmenge an unverständlichen Akronymen, die durch R-ARCSS in den südsudanesischen politischen Diskurs geworfen wird, ein eindeutiges Zeichen für den fehlenden Willen zur Einigung. Jede offene Frage provoziert ein neues Komitee, jeder lose vereinbarte Prozess eine weitere Überwachungs- und Verifizierungskommission. Es ist, als würde eine Transition institutionalisiert, deren einziger Zweck darin besteht, in möglichst komplizierter Art und Weise nicht zu funktionieren. 
Wie dem auch sei. Riek Machar sollte jedenfalls mit der Schar seiner engsten Mitstreiter:innen bis zum 22. Februar in Juba eintreffen. Sonst, so lautete die unausgesprochene Drohung der Regierung, würden die im R-ARCSS getroffenen Vereinbarungen hinfällig. Der Friedensprozess wäre damit de facto beendet. Doch die potenziell negativen Szenarien waren damit keinesfalls durchgespielt.

Chris' Vorsicht gegenüber dem Datum nährte sich primär aus den immer noch lebendigen Erinnerungen an den schon geschilderten Juba Incident vom 8. Juli 2016. An diesem Tag betrat Riek Machar letztmalig den Präsidentenpalast J1. Er verließ ihn nach einem offenen Shoot-Out zwischen seinen Leibwächtern und der präsidentiellen Garde auf der Straße vor dem Palast nach wenigen Minuten fluchtartig wieder. Er zog umgehend mit rund $1.400 \mathrm{Ge}-$ treuen in Richtung der Demokratischen Republik Kongo (DRC). In zum Teil wilden Gefechten verloren in den folgenden vier Tagen über 300 Menschen allein in Juba ihr Leben. Bewaffnete Jugendbanden, vor allem die dem damaligen Generalstabschef Paul Malong, gerüchteweise ohnehin der Mastermind hinter dem Juba Incident (Boswell, 2019: 11), loyal verbundene Dinka-Miliz Mathiang Anyoor, marodierten im Verband mit Armee-Einheiten plündernd durch die Stadt. Es kam zu ethnischen Selektionen und Tötungen.

»Ich werde mein Auto vollgetankt und mit ausreichend Proviant im Hof stehen haben, mit dem Schlüssel im Zündschloss.« Die Frage der Zollbegleichung in Nimule war für Chris um einiges existenzieller, als es den Anschein hatte. Und die Forschungsfrage der Untersuchung, die uns eigentlich nach Bentiu gebracht hatte - die Wahrnehmung des Friedensprozesses im alltäglichen Leben der südsudanesischen Bevölkerung - war in seinem Fall wohl ebenso relevant wie für die von uns zu befragende Zielgruppe im Zuge unserer Forschungsreise. In der Tat ist existenzielle Unsicherheit ein wesentliches verbindendes Element für den überwiegenden Teil der südsudanesischen Bevölkerung. Und nicht nur für die. Auch ich hatte meinen Rückflug im Bewusstsein der Situation für einige Tage vor dem 22. Februar gebucht.

\section{Die politische Transition}

Letzten Endes erweisen sich alle Befürchtungen als unbegründet. Eine Woche vor der Deadline, am 15. Februar 2020, deklariert Salva Kiir, selbst für viele in seinem engsten Umfeld überraschend, die Rückkehr zu den ursprünglichen zehn Gliedstaaten. Nicht verstummen wollende Gerüchte sprechen von 
massivem Druck wichtiger regionaler Verbündeter, insbesondere von ugandischer Seite, der ihn zu diesem Schritt nötigt. Manche von Kiirs engsten Gefolgsleuten haben Mühe, ihre Unzufriedenheit mit der Entscheidung zu verbergen. In der eingeübten Praxis des Gebens und Nehmens am südsudanesischen politischen Marktplatz versucht Kiir, der Entscheidung die Spitze zu nehmen. So werden die zehn Gliedstaaten durch drei Special Administrative Areas ergänzt. Zwei davon sind selbst zwischen den verschiedenen Machtblöcken weitgehend unbestritten.

Abyei, das vom Sudan beanspruchte Territorium nördlich von Warrap, ist ein leicht $\mathrm{zu}$ machendes Zugeständnis an die Ngok Dinka. Auch die von Murle- und Anyuak-Gemeinschaften bewohnte Greater Pibor Administrative Area (GPAA) beruht auf einem schon länger ausgehandelten territorialen Kompromiss. Die GPAA war bereits im Mai 2014 in Folge eines Friedensvertrages der Regierung mit der Cobra Faction, einer von David Yau Yau kommandierten Murle-Miliz, eingerichtet worden. ${ }^{1}$ Yau Yau amtierte als erster Chief Administrator der GPAA, ein Sprungbrett zu einer nationalen politischen Karriere. Selbst wenn die Bedeutung dieser Miliz, ebenso wie das nationale Gewicht von David Yau Yau, unterdessen geschwunden ist, ist die Beibehaltung der GPAA ein wichtiges Signal für den ethnopolitischen Ausgleich in einer notorisch unruhigen Region.

Die dritte Special Administrative Area ringt der SPLM-IO jedoch einiges an Kompromissbereitschaft ab: Ruweng. Bei Ruweng handelt es sich um ein von Padang Dinka bewohntes Gebiet im ursprünglichen Territorium von Unity State, das die in Unity befindlichen Ölfelder an der sudanesischen Grenze mit einschließt. Die Einrichtung einer Ruweng Administrative Area sorgt nicht nur deswegen für massive Unruhe in der Nuer-Bevölkerung. Insbesondere SPLM-IO-Aktivist:innen in Unity sind außer sich vor Wut. Anhand von Karten aus Kolonialzeiten wird versucht, die geografische Legitimität von Ruweng in Frage zu stellen. Wirklich ist Ruweng eine Konsequenz der durch den Bürgerkrieg geschaffenen neuen Realitäten in Unity State. Der im Juni 2020 ernannte neue Chief Administrator, William Chol Awolich, erntet seine politischen Sporen in der Rekrutierung von Padang Dinka für den Kampf gegen die SPLA-IO, vor allem in Abiemnom County nahe Abyei.

Agreement on Resolution of the Conflict in Jonglei State between Covernment of the Republic of South Sudan and South Sudan Democratic Movement/Army-Cobra Faction (SSDM-SSDA-Cobra), unterzeichnet am 9. Mai 2014, https://www.peaceagreements.or g/viewmasterdocument/1675. 
Speziell internationale Beobachter:innen bringen die Ruweng Administrative Area routinemäßig mit der Ölförderung in Verbindung. Es bestehen gute Gründe für diese Annahme. Eine gesicherte physische Kontrolle über das Gebiet um die Explorationsanlagen ist von relevanter Bedeutung. Diese Kontrolle ist durch die mit der Regierungsseite verbündete Padang Dinka-Mehrheit in Ruweng garantiert. Trotz bestehender Konflikte der Padang Dinka mit der Regierung in Juba dürfte die Allianz von dauerhafter Qualität sein.

Als noch wichtiger erscheint jedoch der Aspekt des Machtausgleichs am politischen Marktplatz. Drei Prozent der Einnahmen durch die Ölproduktion sind aufgrund bestehender Kontrakte mit den Explorationsgesellschaften für die Lokalbevölkerung reserviert. Dies ist eine erkleckliche Summe für die Verwaltung der Gliedstaaten und Counties. Dieser Anteil stellt oft die einzige verlässliche Finanzierungsquelle dar, für das offizielle wie das inoffizielle politische Budget. Diese Einnahmen vom Nuer-dominierten Unity State zu den Dinka-Gemeinschaften in Ruweng umzuleiten ist ohne Zweifel hilfreich für Kiir, um die interne, gegen jeden Kompromiss mit der SPLM-IO eingestellte Opposition, wie sie angeblich von hochrangigen Dinka-Persönlichkeiten im Jieng Council of Elders (JCE) betrieben wird, zu befrieden.

Zugleich ist mit der Rückkehr zu den zehn Gliedstaaten die zentrale Bedingung der SPLM-IO für den Einzug in die Regierung erfüllt. Riek Machar erklärt sich nun notgedrungen bereit, seine Position als Erster Vizepräsident im R-TGoNU einzunehmen. »My brother Dr. Riek Machar and I are now partners in peace«, verkündet Salva Kiir in seiner Ansprache bei der Angelobung der neuen Regierung an jenem ominösen 22. Februar. Dennoch ist der Druck, der fraktionsintern auf ihm lastet, merklich:

»I want to appeal to all those who might have been disappointed and angered by the decision on the number of states, to realize that sometimes we have to sacrifice something to gain something even more important. We have sacrificed your states in order to gain peace, which we all desire. We can now discuss in a peaceful environment the states that South Sudanese people wish to have. My entire life and career are built around making compromises to give way to peace and to serve the greater good for our people.«

Die im Friedensvertrag vereinbarte Transitionsregierung ist ein Mammutprojekt. Sie vereint insgesamt fünf Vertragsparteien. Neben der SPLM in Government und jener in Opposition (SPLM-IG und SPLM-IO) inkludiert es eine Gruppe von vormaligen SPLM-Granden, die im Zuge der innerparteilichen Oppositionsregungen gegen Salva Kiir verhaftet wurden, die SPLM-Former 
Detainees (SPLM-FD). Dazu gesellen sich die South Sudanese Opposition Alliance (SSOA), gebildet aus kleineren bewaffneten Milizen, sowie die Gruppe der Other Political Parties (OPP), die sechs zivile politische Stakeholder umfasst. Nachdem sich die militärisch wie politisch dominante SPLM-IG im Zuge der Friedensverhandlungen zu keinen machtpolitischen Kompromissen bereit zeigt, erfordert die Eingliederung der oppositionellen Gruppierungen die Schaffung einer Vielzahl an politisch zu besetzenden Positionen. Schließlich muss gleichzeitig die numerische Dominanz der SPLM-IG gewahrt bleiben.

An jenem 22. Februar wird allerdings zunächst nur die Präsidentschaft besetzt: Salva Kiir wird für die SPLM-IG als Präsident bestätigt, Riek Machar für die SPLM-IO als Erster Vizepräsident eingesetzt. Darüber hinaus werden vier Vizepräsident:innen ernannt. Zwei nominiert die SPLM-IG - Taban Deng Gai, der vormalige Erste Vizepräsident und prominentester Überläufer von der SPLM-IO in die Regierungsfraktion, sowie James Wani Igga, ein langjähriger Kiir-Loyalist ohne starke politische Hausmacht in Central Equatoria (Young, 2019: 30) -, einen die SSOA - Hussein Abdelbagi Akol, ein muslimischer Dinka aus Northern Bahr el-Ghazal. Dazu kommt die Witwe des langjährigen SPLA-Vorsitzenden John Garang, Rebecca Nyandeng De Mabior, die die SPLM-FD repräsentiert.

Die vom R-ARCSS institutionalisierte politische Machtteilung zieht sich allerdings weiter. Sie verläuft durch alle politischen Ebenen, die gesetzgebende Versammlung (das Unterhaus) sowie das Council of States (das Oberhaus), State Governors, State Governments, hin zu den nahezu hundert County Commissioners. Die Stärkeverhältnisse sind eindeutig. Die Transitional National Legislative Assembly (TNLA) wird laut Abkommen mit 550 Parlamentarier:innen bestückt, für ein Land mit etwa zwölf Millionen Einwohner:innen eine stattliche Zahl. 332 davon sollen die SPLM-IG - oder, wie es im Vertrag heißt, die bestehende Übergangsregierung TGoNU - repräsentieren, 128 die SPLM-IO, 50 die SSOA, 30 die OPP, und gerade einmal zehn die SPLM-FD. Ein ähnlicher Prozentsatz wird für die Regierungen auf State und County-Ebene vereinbart: 55 \% für SPLM-IG, $27 \%$ für SPLM-IO, $10 \%$ für SSOA, und 8 \% für OPP.

Dieser Machtteilungskompromiss ist nichts anderes als eine in Zahlen gegossene Machtpolitik, die wenig Zweifel daran lässt, wer den südsudanesischen Bürgerkrieg de facto für sich entschieden hat. Daran ändert die zusätzlich vereinbarte Frauen-Quote von 35 \%, die auf allen Ebenen der Ernennungen einzuhalten ist, wenig. Angesichts der Zahlenverhältnisse sollte es 
der Regierungsfraktion sogar deutlich leichter fallen, diese Quote zu erfüllen. Die deutlich größere Zahl an zur Verfügung stehenden Posten sollte doch einen um einiges größeren Bewegungsspielraum ermöglichen. Dies bleibt jedoch Wunschdenken. Zum Zeitpunkt des Schreibens dieses Buches ist die Besetzung vieler Sitze und Positionen noch nicht abgeschlossen. Aufgrund der schon vorgenommenen Ernennungen ist es aber wahrscheinlich, dass die Frauen-Quote nonchalant auf dem Altar der innerfraktionellen Machtpolitik geopfert wird.

Eine der impliziten Grundannahmen der im Friedensvertrag vereinbarten politischen Machtteilung ist, dass die Einrichtung einer gemeinsamen Regierung zu einer Überwindung der durch den Bürgerkrieg aufgerissenen Gräben beiträgt. Eine oberflächliche Kenntnis der Geschichte könnte diese Annahme bestätigen. Schließlich waren die allermeisten der im R-TGoNU engagierten Protagonist:innen einmal, in unterschiedlichsten Funktionen, Mitglied einer SPLA/M. Die ersten Ernennungen spiegeln die Idee einer kollaborativ angelegten politischen Machtteilung wider. So erhält die Opposition einige nominell relevante Positionen.

Das Verteidigungsministerium wird mit Angelina Jany Teny besetzt, einer prominenten und, speziell in Unity State, überaus populären Protagonistin der SPLM-IO, sowie Ehefrau von Riek Machar. Auch der Ölminister wird von der SPLM-IO nominiert. Der aktuelle Minister, Puot Kang Chuol, gilt als einer von Machars engsten Vertrauten. Trotz der Besetzung einiger formeller Schlüsselministerien durch Vertreter:innen der Opposition bleibt die reale Machtbalance in der Regierung gewahrt. Angelina Teny zur Verteidigungsministerin zu machen ist eine Sache, ihr eine konkrete Befehlsgewalt gegenüber den von SPLM-IG kontrollierten Truppenteilen einzuräumen, die diese noch dazu akzeptieren würden, eine andere.

Ein weiteres der von der SPLM-IO besetzten Ministerien ist das Ministry of Peacebuilding. Stephen Par Kuol, ein Gawaar Nuer aus Jonglei, übernimmt die Position. Stephen Par ist ein langjähriger Verbündeter von Riek Machar, ein Bündnis, das noch auf Zeiten des Nasir-Splits im Jahr 1991 zurückgeht. Später, während des südsudanesischen Bürgerkrieges, fungiert Stephen Par als Leiter von Machars Diaspora-Netzwerk in den USA und in Kanada. Seine faktische Situation in der Regierung ist allerdings prekär. Er ist ein Minister ohne Ministerium. Er residiert in einem kleinen Sitzungszimmer eines Hotels, ohne Internet, phasenweise ohne Strom.

Stephen Par ist engagiert und zweifellos gewillt, seinem Ministerium Gewicht zu verleihen. Er hat akzeptiert, dass er in seiner Rolle den nationa- 
len Friedensprozess nicht beeinflussen kann. Die R-ARCSS-Transition liegt unzweifelhaft außerhalb der Kompetenz eines noch dazu die Opposition repräsentierenden Peacebuilding-Ministers. Stephen Pars Interesse gilt daher den subnationalen und lokalen Konflikten, in Jonglei, in Lakes, in den Equatorias. In seinen optimistischen Momenten plant er die Einrichtung von Peacebuilding-Ministerien in allen State Governments und den Ausbau seines Ministeriums zur zentralen Anlaufstelle des südsudanesischen Peacebuilding.

Die Realität ist ein kleiner Sitzungssaal in einem alles andere als luxuriösen Hotel, das derzeit als Ministerium fungiert. In anderen Hotelzimmern war während überlanger Frist das NPTC, das National Pre-Transition Committee, untergebracht. Zumindest ist mittlerweile das $» \mathrm{P}$ « aus dem Namen verschwunden, untrügerisches Zeichen dafür, dass die formelle Transitionsphase eingeleitet wurde. Was nach wie vor fehlt, ist Strom und Internet. Ein Gecko läuft an der Wand hinter dem Minister seine Runden. Bei unserem Gespräch hat es weit über $30 \mathrm{Grad}$, wir alle schwitzen - Chris, ich und unser Begleiter von der UN mit Covid-19-bedingtem Gesichtsschutz, der Minister ohne. Die SPLM-IO-Teile der Regierung befinden sich offenkundig in einer schwierigen Situation. Und auf der Suche nach internationaler Unterstützung. Der Minister war gerade zuvor kurzfristig zu einer Besprechung nach J1 - dem Präsidentenpalast - gerufen worden. Sein Office Manager hatte unser Treffen schon offiziell abgesagt, da kommt der Minister doch. Er hatte eine Lücke im Terminkalender gefunden. Dies lässt wenig Zweifel daran, dass ein Treffen mit internationalen Partnern für ihn zumindest ebenso viel Sinn macht wie ein Treffen mit dem Präsidenten. Bei Salva Kiir ist für IO-Minister:innen wenig zu gewinnen.

Das Ministry of Peacebuilding besteht zu diesem Zeitpunkt aus zwei Herren. Neben Stephen Par, dem Minister, und seinem Office Manager, der zuvor in einer humanitären INGO gearbeitet hat. Der Office Manager lässt keinen Zweifel daran, dass ihn seine Aufgabe im Ministerium reizt und er sie im Griff hat. Politisches Ins-Abseits-Stellen ist für ihn höchstens eine Herausforderung. Das Problem liegt woanders. Es ist Geld. Derzeit fungiert das UNDP als der einzige wirkliche Geldgeber des Ministeriums. Das reicht nicht einmal für die gegenwärtig limitierten Tätigkeiten. Und selbst der leitende Ministeriumsangestellte muss sich fragen, wie er Ausgaben wie etwa das Schulgeld seiner Kinder von seinem Gehalt bezahlen kann. Auf längere Sicht ist das kaum möglich. 
Eine politische Machtteilung in Friedensprozessen ist so gut wie nie auf eine faire Verteilung politischer Güter zurückzuführen. Es geht um Brokerage. Christine Bell und ich haben für derartige Konstellationen, die durch Friedensprozesse herbeigeführt und institutionalisiert werden, den Begriff des »Formalised Political Unsettlement « geprägt (Bell und Pospisil, 2017). Allzu oft wird die Unterzeichnung eines Friedensvertrages mit einem tatsächlichen Willen der Konfliktparteien zu einem genuinen Friedensschluss verwechselt. In der Realität geht es allerdings zumeist einzig um eine Formel der Machtteilung, die den Parteien die Option einer nicht-bewaffneten Weiterführung des politischen Konfliktes - oder dessen Einfrieren - schmackhaft macht oder überhaupt politisch ermöglicht. Beispiele für die idealisierte Vorstellung, wonach solche Machtteilungskompromisse langfristig zu einer politischen Normalisierung oder gar zu einer demokratischen Polity führen würden, finden sich in der empirischen Realität von Konflikt-Transitionen kaum.

Ein Fall, der diese Schwierigkeit wie kaum ein anderer verdeutlicht, ist das im Jahr 1995 geschlossene Dayton-Friedensabkommen für Bosnien und Herzegowina. Das Abkommen soll nicht nur das Ende des Bosnien-Krieges markieren, sondern den Startpunkt für eine demokratische Transition des Landes setzen. Die ethnopolitisch verankerte und international überwachte Machtteilung zwischen den beiden bosnisch-herzegowinischen Entitäten, der Bosnisch-Kroatischen Föderation und der Republika Srpska, ist allerdings ein Vierteljahrhundert nach dem Friedensschluss nach wie vor unverändert in Kraft. Postenbesetzungen erfolgen auf Basis ethnopolitischer Kriterien, öffentliche Karrieren, die ohne Ansicht der Person einzig auf Fähigkeiten und erbrachten Leistungen basieren, sind nach wie vor unmöglich. Anzeichen einer langfristigen politischen Normalisierung in Richtung eines demokratischen Rechtsstaates sind der Akzeptanz der Verstetigung der ursprünglich als vorübergehend gedachten Struktur der Machtteilung gewichen.

Auch der R-ARCSS repräsentiert ein solches »Formalised Political Unsettlement« in einer fast schon prototypischen Form. Die SPLM-IG wählte gemäß ihrem Selbstverständnis als militärische Gewinnerin des Bürgerkrieges den Weg des politischen Kompromisses aus primär taktischen Erwägungen, nicht zuletzt aufgrund massiven regionalen politischen Drucks. Für die anderen unterzeichnenden Gruppierungen war die Machtteilungsformel des RARCSS das zum gegebenen Zeitpunkt bestmögliche politische Resultat eines immer schwieriger werdenden militärischen Kampfes. Von einer genuinen Einsicht in potenzielle gemeinsame Interessenlagen oder gar in übergeord- 
nete Interessen wie solche des Nationalstaates oder seiner Bewohner:innen waren alle Seiten weit entfernt. Von daher überrascht die harzige Umsetzung der Machtteilungsformel und der faktische Ausschluss der vormaligen Opposition durch die vormalige Regierungsfraktion wenig.

Was wäre die Alternative $\mathrm{zu}$ einem »Formalised Political Unsettlement" gewesen? Die Besetzungen der Positionen gestalten sich schwierig, der Prozess ist mittlerweile um viele Monate verspätet. Aber der Waffenstillstand, der mit der Unterzeichnung von R-ARCSS zwischen den Vertragsparteien vereinbart wurde, hält - selbst in Anbetracht einer Anzahl von gröberen Verstößen in der zweiten Jahreshälfte des Jahres 2020. Und das ist letztendlich die größte Leistung, die R-ARCSS abzuliefern imstande war. Der Vertrag liefert allen Fraktionen in erster Linie eine Entschuldigung, den bewaffneten $\mathrm{Ab}$ nützungskampf gegeneinander nicht weiter fortzusetzen. Angesichts eines $\mathrm{zu}$ diesem Zeitpunkt bereits sieben Jahre andauernden Bürgerkrieges ist dies eine nicht zu vernachlässigender Errungenschaft.

Die konkrete Umsetzung der Machtteilung ist voller Fallstricke. Nach längeren Verhandlungen ernennt Salva Kiir Ende Juni 2020 die ersten acht der zehn State Governors. Zwei Wochen später wird der von SSOA nominierte Denay Jock Chagor, der vormalige Stellvertreter des 2019 verstorbenen SPLAGenerals und nachmaligem Bul Nuer-Warlord Peter Gadet, als Gouverneur von Jonglei eingesetzt. Die Besetzung des Gouverneurs von Upper Nile State, dem Gliedstaat im äußersten Nordosten des Landes, gerät zum substanziellen Zankapfel. Entlang der Vereinbarungen in R-ARCSS steht das Vorschlagsrecht für den State Governor von Upper Nile der SPLM-IO zu. Diese macht davon Gebrauch und nominiert Johnson Olonyi als ihren Kandidaten, was Salva Kiir wiederum mit einer Kaskade an Scheinargumenten zurückweist. Olonyi wäre im Ausland und könnte daher nicht ernannt werden (was bei anderen Ernennungen keine Rolle spielte). Olonyi wäre nach wie vor in aktive Kampfhandlungen verstrickt. Zudem würde die Bevölkerung von Upper Nile Olonyi als Gouverneur ablehnen.

Das Dickicht an Interessen hinter dieser Ernennung zu entwirren ist vermutlich selbst für unmittelbar Involvierte eine herausfordernde Aufgabe. Einige der Aspekte näher zu beleuchten eröffnet jedoch einen nüchternen Blick auf die politische Dimension der Transition. Sie setzt die plakative Fokussierung auf die Handlungen der beiden nach außen sichtbaren Protagonisten Kiir und Machar in ihren notwendigen strukturellen Rahmen. Johnson Olonyi ist keine austauschbare Figur im komplexen Machtgefüge von Upper Nile. Seine politische Geschichte beginnt mit der Abspaltung des ehemaligen 
Außenministers des Sudan und prominenten Shilluk-Politikers, Lam Akol, im Jahr 2009. Er formiert unter dem Namen SPLM-Democratic Change (SPLM-DC) eine von der SPLM/A unabhängige Formation mit dem Ziel, eigenständig an den letzten Wahlen des noch vereinigten Sudan teilzunehmen. Im gesamtsudanesischen Parlament erreicht die SPLM-DC zwei der insgesamt 450 Sitze (die SPLM deren 99). In der Wahl zum südsudanesischen Übergangspräsidenten kann Lam Akol knapp 200.000 Stimmen, ein Anteil von rund 7\%, auf sich vereinigen. Er tritt als einziger Gegenkandidat von Salva Kiir an, der die Wahl mit $93 \%$ der Stimmen haushoch für sich entscheidet.

Was bei einem flüchtigen Blick auf die Zahlen wie ein demokratischer Prozess erscheinen könnte, verläuft in Upper Nile State, in dem sich die Siedlungsgebiete der Shilluk befinden, alles andere als gewaltfrei. Die SPLA, die die SPLM-DC der Kollaboration mit Khartum verdächtigt, verhaftet einige der neu gewählten Shilluk-Politiker. Darauffolgende Proteste werden mit Waffengewalt angegriffen. Insbesondere in Fashoda County nördlich von Malakal eskalieren die Kämpfe, als sich Shilluk-Milizen unter dem Kommando von Robert Gwang Nyikier Yur der SPLA entgegenstellen. Bald bekommen diese Kämpfe eine ethnopolitische Dimension. Die SPLA-Einheiten werden als Schutztruppen der Padang Dinka wahrgenommen und greifen in den Auseinandersetzungen auf Padang Dinka-Milizen zurück. ${ }^{2}$

In diesem Kontext nimmt Johnson Olonyis politische Karriere ihren Ausgang. Die Angriffe der SPLA zerstören die Geschäfte des mittelständischen Unternehmers. Olonyi schließt sich Gwangs Miliz an und erwirbt sich schnell den Ruf eines unerschrockenen, fähigen und entschlossenen Militärführers. Er wird Gwangs Stellvertreter und übernimmt Teile von dessen Miliz, nachdem sich Gwang im August 2010 dafür entscheidet, sich wieder in die SPLA, seiner ehemaligen politisch-militärischen Heimat, einzugliedern. Olonyi entwickelt sich zu einem respektierten Warlord. Vor allem seine erfolgreichen Kämpfe gegen die von Nuer dominierte 7. Division der SPLA, die einst unter dem Kommando des bereits erwähnten, legendären Warlords Peter Gadet standen, machen ihn unter militanten Shilluk populär.

Der Ausbruch des Bürgerkrieges im Jahr 2013 mischt die Karten neu. Nach der Abspaltung der SPLA-IO entscheidet sich Olonyi zunächst für die Regierungsseite und kämpft mit seiner zu diesem Zeitpunkt etwa 3.000 Mann star-

2 Vgl. die Nachzeichnung des Konfliktes in einem Situational Update von Small Arms Survey, www.smallarmssurveysudan.org/facts-figures/south-sudan/conflict-of2013-14/the-conflict-in-upper-nile.html (22-12-2020). 
ken Agwelek-Miliz überaus erfolgreich gegen SPLA-IO-Einheiten am westlichen Nil-Ufer. Allerdings ist sogar unter seinen wechselnden politischen Freunden unbestritten, dass es sich bei Olonyi um einen untypischen Spieler am südsudanesischen politischen Marktplatz handelt. Seine politische Loyalität definiert sich in erster Linie ethnopolitisch.

Nach aufkommenden Konflikten mit den von Padang Dinka aus Ruweng dominierten SPLA-Einheiten in Upper Nile wechselt er prompt die Seiten. Doch als Teil der SPLA-IO bekommt sein Image als erfolgsverwöhnter Warlord Risse. Nun selbst in der Verteidigungsposition am westlichen Nil-Ufer, muss Olonyi schwere militärische Schläge einstecken. Ohnehin ist die Region durch den Krieg zerstört. Malakal, die Hauptstadt von Upper Nile State, ist nach zwölfmaligem Wechsel der militärischen Kontrolle weitgehend dem Erdboden gleichgemacht. Der Großteil der Bevölkerung hat sich in das von UNMISS geschützte PoC-Camp zurückgezogen. Olonyi kann der Vertreibung der Shilluk-Gemeinschaften durch die SPLA wenig entgegensetzen. Er zieht sich aus der Region zurück. Ab dem Jahr 2017 ist er vermehrt in Khartum anzutreffen, was Gerüchte über seine Kollaboration mit dem NCP-Regime unter Bashir aufkommen lässt.

Diese Geschichte gibt einen Einblick in die vielschichtigen Konsequenzen von Olonyis Nominierung als State Governor. Für die SPLM-IO repräsentiert Olonyi ihren vielleicht wichtigsten Verbindungspunkt zu den ShillukGemeinschaften, ohne deren Unterstützung im ölproduzierenden Upper Nile State wenig zu gewinnen ist. Zugleich gilt Olonyi als ein fraktionsinterner Kritiker von Machars Führungsposition in der SPLM-IO. Das eröffnet Machar, unter dem beständigen Ruch der Günstlingswirtschaft stehend, die Chance, mit der Ernennung plural und gegenüber seinen organisationsinternen Kritiker:innen inklusiv zu erscheinen.

Gleichzeitig setzt die Nominierung Salva Kiir unter substanziellen Druck. Ohnehin fühlen sich die im Osten des Südsudan von Jonglei bis Upper Nile siedelnden Dinka-Gemeinschaften in Hinblick auf die SPLM/A-interne Machtverteilung gegenüber den Bahr el-Ghazal Dinka fortwährend benachteiligt. Nun sollte Upper Nile an einen ihrer deklarierten Erzfeinde gehen? Glaubwürdige Erzählungen berichten von harschen Einflussnahmen auf Kiir, dem offenbar vermittelt wird, dass die Dinka-Gemeinschaften in der Region eine Ernennung Olonyis als eine Kriegserklärung auffassen würden. Zugleich lässt Olonyi über lange Zeit kein Zeichen der Schwäche erkennen. Eine Presseerklärung der SPLM-IO verweist Anfang Dezember 2020 keck darauf, dass er keine Sicherheitsgarantien benötige, würde er doch zu seiner 
Ernennung in Juba mit seinem eigenen militärischen Personal anreisen. Da ist sie wieder, die Erinnerung an den Juba Incident von Juli 2016.

Die Antwort von Salva Kiir auf diese unangenehme Situation ist nur dann außergewöhnlich, wenn die für die erfolgreiche Operation am politischen Marktplatz unabdingbare Fähigkeit zu strategischem Pragmatismus unterschätzt wird. Denn entgegen der nach außen hin martialischen Rhetorik ist Kiir keineswegs bemüht, Olonyi ein für alle Mal von den Trögen der Macht fernzuhalten. Die Konfrontation ist in keiner Weise persönlich. Vielmehr geht es um die Verhandlung um den geeigneten Modus für Olonyis Integration. Und die Position als Gouverneur scheint dafür nicht geeignet.

Ein Kader der SPLM-IO aus Upper Nile muss Ende des Jahrs 2020 ob meiner Nachfrage, was Kiir gegen Olonyi einzuwenden hätte, schmunzeln. Gegen ihn einzuwenden? »Salva ruft ihn jeden Tag an, um ihn zum Absprung zu bewegen. Er will, dass Johnson zur SPLM-IG zurückkehrt«, meinte er. Rückfragen bei anderen, für gewöhnlich gut informierten Kolleg:innen bestätigten diese Erzählung. Kiirs erste Option eines Auswegs aus der verfahrenen Situation ist der klassische Schachzug eines gekonnten Spielers am politischen Marktplatz: der Einkauf von politischer Loyalität, der den Gegenpart belohnt und zugleich strategisch aus dem Spiel nimmt.

Die letztendliche Lösung kommt für nahezu alle Akteure unerwartet. In einer überraschenden Einigung Ende Januar 2021 weicht Riek Machar von Olonyis Nominierung ab und verständigt sich mit Salva Kiir auf die Ernennung von Budhok Ayang Nai Kur. Eigentlich gilt Budhok Ayang als enger Vertrauter von Johnson Olonyi. Dieser zeigt sich allerdings in ersten Reaktionen von seiner Kaltstellung alles andere als begeistert. Die Karten in Upper Nile und zum Teil auch innerhalb der SPLM/A-IO - sind jedenfalls neu gemischt. Die Zukunft wird zeigen, was die frisch zusammengestellte Konfiguration bewirkt.

Niemals wird sich herausfinden lassen, ob Machar ernsthaft mit Olonyis Bestätigung durch die Präsidentschaft gerechnet hat. Das Eintauchen in die Spezifika dieser Personalie dient aber einem anderen Zweck. Johnson Olonyi ist nur einer von vielen eher marginal positionierten Spieler:innen auf dem südsudanesischen politischen Marktplatz. Und dennoch gestaltet sich seine Ernennung als ein hochkomplexer Prozess, der Elemente seiner Biografie, seiner ethnopolitischen Identität und territorialen Verankerung, seiner militärischen Handlungsmacht und seiner wechselnden politischen Loyalitäten mit einschließt. Sicherlich ist nicht jede Personalie gleich spektakulär. Allerdings zeigt das Beispiel Olonyi die Tiefe der an der Oberfläche so einfach 
erscheinenden Zahlen und Prozentsätze der Ernennungen entlang der im RARCSS festgelegten Formel der politischen Machtteilung.

Soundso viel Prozent für diese, soundso viel Prozent für jene, und $35 \%$ aller Ernennungen Frauen. Dahinter liegt eine um ein Vielfaches komplexere Formel, die, um tragfähig zu sein, einen komplexen und vielschichtigen Interessenausgleich gewährleisten muss, dessen Gestaltung nirgends festgeschrieben ist oder überhaupt festgeschrieben werden kann. Mathematisch unbestimmbar ist die Gewährleistung einer haltbaren Machtbalance eine Sache des Gefühls und der permanenten Aushandlung. Das Austarieren dieser Balance ist ohne Zweifel die Meisterschaftsdisziplin jener Protagonist:innen am politischen Marktplatz, die dessen permanente Fragilität über lange Jahre und Jahrzehnte nicht nur bestimmen, sondern auch politisch sowie physisch heil überstehen. Nur wenige sind in der Lage, diesen feinen Interessenausgleich über ausgedehnte Zeiträume zu verwalten und ihn selbst in Phasen der Turbulenz, wie sie der Prozess der Postenbesetzungen in der Folge eines Friedensabkommens darstellt, weiterzuführen.

Das Prinzip der politischen Machtteilung wurde nicht mit dem R-ARCSS eingeführt. Vielmehr war der politische Machtausgleich von Beginn an in das System der SPLM/A eingeschrieben, einschließlich all seiner Volatilität und Prekarität. Eine Transition aus diesem System zu ermöglichen, wie es die Neuerrichtung einer verfassungsmäßigen Ordnung im R-ARCSS vorsieht, ist kein einfacher Prozess, der sich mit einem einmaligen Wahlprozess institutionell realisieren ließe.

\section{Sicherheitssektorreform}

Die Besetzung der Positionen in Exekutive und Legislative hat die Militarisierung der Politik im Südsudan bereits eindrücklich vor Augen geführt. Wenig überraschend ist es daher die Sicherheitssektorreform (SSR), der von den meisten nationalen wie internationalen Beobachter:innen eine Schlüsselrolle beim Erfolg der südsudanesischen Transition zugeschrieben wird. Tatsächlich ist der Sicherheitssektor der ausschlaggebende Faktor für politische Autorität auf nationaler Ebene. Ob sich der Sektor angesichts seiner besonderen Bedeutung für eine tiefergreifende Reform offen zeigt, ist, gelinde gesagt, umstritten. In jedem Fall ist es eine hochbrisante Problematik.

Trotz der offiziellen Unterstützung der im R-ARCSS vereinbarten Festlegungen durch internationale Partner ist die SSR-Thematik eine Materie, an 